\title{
Perpendicular Magnetocrystalline Anisotropy in Tetragonally Distorted Fe-Co Alloys
}

\author{
Gabriella Andersson, ${ }^{1, *}$ Till Burkert, ${ }^{2}$ Peter Warnicke, ${ }^{3}$ Matts Björck, ${ }^{1}$ Biplab Sanyal, ${ }_{1}^{1}$ Cyril Chacon, ${ }^{1}$ Claudia Zlotea, ${ }^{4}$ \\ Lars Nordström, ${ }^{1}$ Per Nordblad, ${ }^{3}$ and Olle Eriksson ${ }^{1}$ \\ ${ }^{1}$ Department of Physics, Uppsala Universitet, Box 530, 75121 Uppsala, Sweden \\ ${ }^{2}$ IFM, Linköpings Universitet, SE-581 83 Linköping, Sweden \\ ${ }^{3}$ Department of Engineering Sciences, Uppsala Universitet, Box 534, 75121 Uppsala, Sweden \\ ${ }^{4}$ Department of Materials Chemistry, Uppsala Universitet, Box 538, 75121 Uppsala, Sweden
}

(Received 7 September 2005; published 27 January 2006)

\begin{abstract}
We report on the experimental realization of tetragonal Fe-Co alloys as a constituent of $\mathrm{Fe}_{0.36} \mathrm{Co}_{0.64} / \mathrm{Pt}$ superlattices with huge perpendicular magnetocrystalline anisotropy energy, reaching $210 \mu \mathrm{eV} / \mathrm{atom}$, and a saturation magnetization of $2.5 \mu_{\mathrm{B}} /$ atom at $40 \mathrm{~K}$, in qualitative agreement with theoretical predictions. At room temperature the corresponding values $150 \mu \mathrm{eV} /$ atom and $2.2 \mu_{\mathrm{B}} /$ atom are achieved. This suggests that Fe-Co alloys with carefully chosen combinations of composition and distortion are good candidates for high-density perpendicular storage materials.
\end{abstract}

The enormous increase in the recording density of hard disk drives, by more than 6 orders of magnitude during the past 50 years, has mainly been achieved by simply scaling the dimensions of the bits recorded in the storage layer [1]. However, this traditional scaling is limited by the onset of superparamagnetism. This occurs when the grain volume $V$ in the recording medium is reduced so that the ratio of the magnetic energy per grain to the thermal energy, $K_{\mathrm{u}} V / k_{\mathrm{B}} T$, becomes sufficiently small to cause the recorded data to be erased by thermal fluctuations in an intolerably short time [1,2]. $K_{\mathrm{u}}$ is the uniaxial magnetocrystalline anisotropy energy (MAE), i.e., the energy required for rotating the magnetization direction from an easy axis to the hard axis. Thus, high- $K_{\mathrm{u}}$ materials [3] are needed to further increase the recording density. The maximum practical MAE, however, is limited by the required write field $H_{\mathrm{w}} \approx K_{\mathrm{u}} / M_{\mathrm{s}}$, which has to be delivered by the writing head. Thus, a large value of $M_{\mathrm{s}}$, the saturation magnetization of the recording medium, will be beneficial both through decreasing $H_{\mathrm{w}}$ as well as by increasing the field available in the readback process. Hence, large values of $K_{\mathrm{u}}$ and $M_{\mathrm{s}}$ are indispensable properties of future high-density magnetic recording materials.

Recently, based on first-principles calculations, tetragonal $\mathrm{Fe}-\mathrm{Co}$ alloys were proposed as promising materials that combine the desired large values of $K_{\mathrm{u}}$ and $M_{\mathrm{S}}$ [4]. The advantages of the suggested alloys, as compared to other materials considered for magnetic storage [3], are their about 50\% larger saturation magnetization, the huge perpendicular MAE, and the possibility to tailor the MAE by changing the alloy concentration. In addition, Fe-Co alloys do not require as high deposition temperatures as, e.g., chemically ordered $\mathrm{L} 1_{0} \mathrm{FePt}$ [5], which has received considerable attention recently. From the calculations it was found that, for certain values of the ratio $c / a$, between the lengths of the body-centered tetragonal (bct) crystal's $c$ and $a$ axes, and for specific alloy concentrations, very high values of $K_{\mathrm{u}} \approx 800 \mu \mathrm{eV} /$ atom can be expected. This MAE, which is larger by 3 orders of magnitude than for bcc Fe, occurs theoretically for a composition of about $\mathrm{Fe}_{0.4} \mathrm{Co}_{0.6}$ and $c / a=1.20-1.25$. Also, the predicted easy axis of magnetization for the tetragonal alloy is along the $c$ axis, which facilitates the usage in perpendicular magnetic recording applications.

The mechanism responsible for this giant MAE has been identified by an analysis of the electronic states at the $\Gamma$ point at the center of the Brillouin zone, which govern the MAE of tetragonal $\mathrm{Fe}[4,6]$. When the cubic symmetry is broken through tetragonal distortion, electronic states that are degenerate in the cubic state will split and cross other states when the $c / a$ ratio is around 1.2. From a second order perturbation treatment of the MAE it follows that a strongly enhanced $K_{\mathrm{u}}$ can be expected if the alloy concentration is chosen such that the Fermi energy is at the band crossing $[4,6]$.

It is important to note that the proposed bct structure gradually transforms into the face-centered cubic (fcc) structure as $c / a$ approaches $\sqrt{2}$. It has been observed that very thin Fe layers have fcc structure in $\mathrm{Fe} / \mathrm{Pt}$ superlattices [7-9]. In this Letter the parameters $c_{\mathrm{FeCo}}$ and $a_{\mathrm{FeCo}}$ of the $\mathrm{Fe}-\mathrm{Co}$ alloy refer to the bct structure. Note also that for corresponding tetragonal structures of pure $\mathrm{Fe}$ and $\mathrm{Co}$, the easy magnetization axis is in the basal plane [6].

One way of manufacturing $\mathrm{Fe}-\mathrm{Co}$ alloys with tetragonally distorted structure is by epitaxial growth in conjunction with another material in a superlattice. The second material used in the growth of the superlattice is required to have, apart from the ability to grow in a layer-by-layer mode with the Fe-Co alloy, an equilibrium atomic volume such that the $\mathrm{Fe}-\mathrm{Co}$ unit cell is contracted in the plane, resulting in a bct structure with $c / a>1$. For the present study $\mathrm{Pt}$ was chosen, since the growth of $\mathrm{Fe} / \mathrm{Pt}$ and $\mathrm{Co} / \mathrm{Pt}$ superlattices with promising $c / a$ ratios is well established [7-11]. The resulting $c / a$ can be tailored, within a certain 
range, by adjusting the ratio of the $\mathrm{Fe}-\mathrm{Co}$ and $\mathrm{Pt}$ layer thicknesses.

The growth of a series of samples, with varied $c / a$ ratio in the $\mathrm{Fe}-\mathrm{Co}$ layers, was performed by dc magnetron sputtering in an ultrahigh vacuum system, with a base pressure below $1 \times 10^{-7} \mathrm{~Pa}\left(1 \times 10^{-9}\right.$ torr $)$, using argon $(0.8 \mathrm{~Pa})$ as a sputter gas. A buffer layer of $0.6 \mathrm{~nm} \mathrm{Fe}$ and $3.9 \mathrm{~nm} \mathrm{Pt}$ was deposited onto a $\mathrm{MgO}(001)$ substrate at $600 \mathrm{~K}$ [7] to minimize the lattice mismatch between superlattice and substrate. Then, typically $20-25$ bilayers of $\mathrm{Fe}-\mathrm{Co}$ and Pt were deposited onto the buffer at $470 \mathrm{~K}$. The alloy was created by $\mathrm{Co}$ sputtering individual $\mathrm{Fe}$ and $\mathrm{Co}$ targets, the composition being controlled through the electric power on the magnetrons. The final concentrations were determined by Rutherford backscattering spectrometry (RBS) and x-ray photoelectron spectroscopy (XPS) to be (36 \pm 5$)$ at. \% $\mathrm{Fe}$ and $(64 \pm 5)$ at. \% Co. In the following, "FeCo" will refer to this $\mathrm{Fe}_{0.36} \mathrm{Co}_{0.64}$ alloy. The relatively large uncertainty arises, in the case of RBS, from the fact that $\mathrm{Fe}$ and Co have similar masses, and for XPS, being the more accurate method for the present samples, on the calibration using target pieces of pure $\mathrm{Fe}$ and $\mathrm{Co}$ as references.

This Letter will focus on the superlattice with 23 bilayers, each consisting of $3 \mathrm{ML}$ (monolayers) of Fe-Co and $7 \mathrm{ML}$ of Pt. A schematic view, with ideal interfaces, of this particular sample is shown in Fig. 1. Further details on the growth and characterization of the extended sample series will be published elsewhere [12]. It is, however, appropriate to account for the structural properties of this " $3 / 7$ " sample: interatomic distances, crystalline quality, and

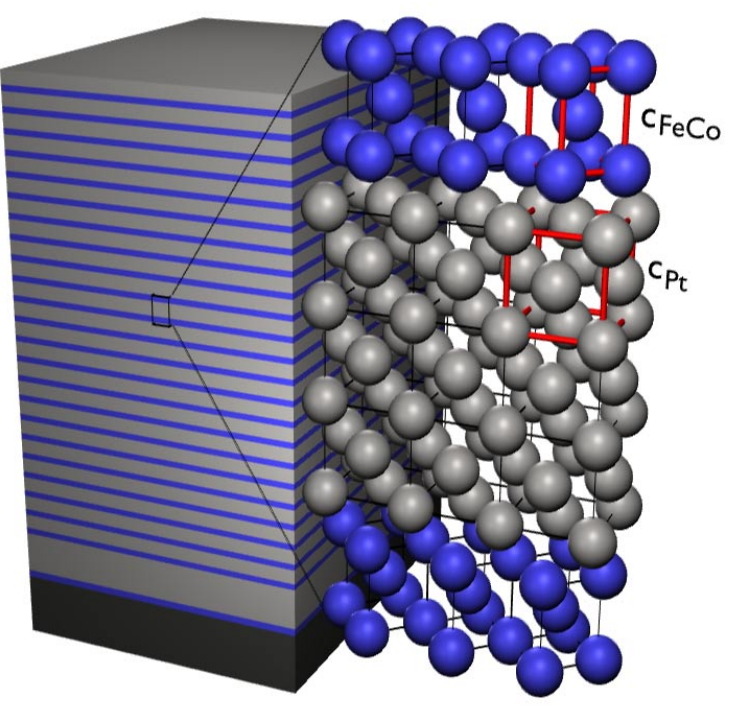

FIG. 1 (color online). Schematic picture of the " $3 / 7$ " superlattice in the present study. The individual perpendicular lattice parameters, $c_{\mathrm{FeCo}}$ and $c_{\mathrm{Pt}}$, are defined in the enlarged cut. The unit cells of Pt and Fe-Co have in-plane base edges $a_{\mathrm{Pt}}$ and $a_{\mathrm{FeCo}}$, respectively, and are rotated with respect to each other by $45^{\circ}$. well-defined layered character, as investigated by $\mathrm{x}$-ray reflectivity, diffraction (XRD), and reciprocal space mapping (RSM). In the discussion on crystal structure below, the superlattice [100] and [010] directions are defined by the substrate edges, i.e., the $\mathrm{MgO}$ and $\mathrm{Pt}$ [100] and [010] directions. The $45^{\circ}$ rotation between the $\mathrm{FeCo}$ and $\mathrm{Pt}$ inplane axes is described in Fig. 1. It is assumed that both constituents adapt the same interatomic distance in the (001) plane.

The results from the XRD and reflectivity measurements are presented in Fig. 2. Using the width of the XRD peak labeled " $\mathrm{FeCo} / \mathrm{Pt}(002)$ " in the Scherrer formula [13] gave a minimum perpendicular structural coherence length of $20 \mathrm{~nm}$, corresponding to $10-11$ bilayer repetitions. The fine oscillations in the reflectivity data, seen in the inset in Fig. 2, show that the total thickness is well defined.

Considering that the Pt layers are thicker than the FeCo layers, the best approach to deriving a $c_{\mathrm{FeCo}}$ value is through eliminating a reasonable $c_{\mathrm{Pt}}$ from the experimental perpendicular lattice parameter $\langle c\rangle$, which is a weighted average of $c_{\mathrm{Pt}}$ and $c_{\mathrm{FeCo}}$ [14]. From the $\mathrm{FeCo} / \mathrm{Pt}(002)$ peak in Fig. 2 the parameter $\langle c\rangle=0.3876 \mathrm{~nm}$ was obtained. The in-plane lattice parameter, $a_{\mathrm{FeCo}} \sqrt{2}=a_{\mathrm{Pt}}=0.379 \mathrm{~nm}$, was obtained from RSM scans around the (002) and (113) Bragg peaks of the superlattice [13], yielding $a_{\mathrm{FeCo}}=0.268 \mathrm{~nm}$ in the bct alloy. Then a value $c_{\mathrm{Pt}}=$ $0.417 \mathrm{~nm}$ was obtained from the strained $a_{\mathrm{Pt}}$, assuming a volume-preserving elastic response of the Pt fcc unit cell [15]: $\Delta c / c_{0} \approx-2 \Delta a / a_{0}$. This assumption is justified by a structural relaxation performed using an electronic band structure method, which will be described below and where the Pt volume change is negligible. The value agrees with the $0.416 \mathrm{~nm}$ obtained from XRD on the somewhat strained $\mathrm{Pt}$ buffer layer. Finally the $\mathrm{FeCo}$ parameter $c_{\mathrm{FeCo}}=0.317 \mathrm{~nm}$, i.e., $c / a \approx 1.18$, was derived from $\langle c\rangle=0.3876 \mathrm{~nm}, \quad c_{\mathrm{Pt}}=0.417 \mathrm{~nm}, \quad$ and the relative amounts of $\mathrm{FeCo}$ and $\mathrm{Pt}$ in the bilayers. The latter were determined from simulations of the $\mathrm{x}$-ray reflectivity using

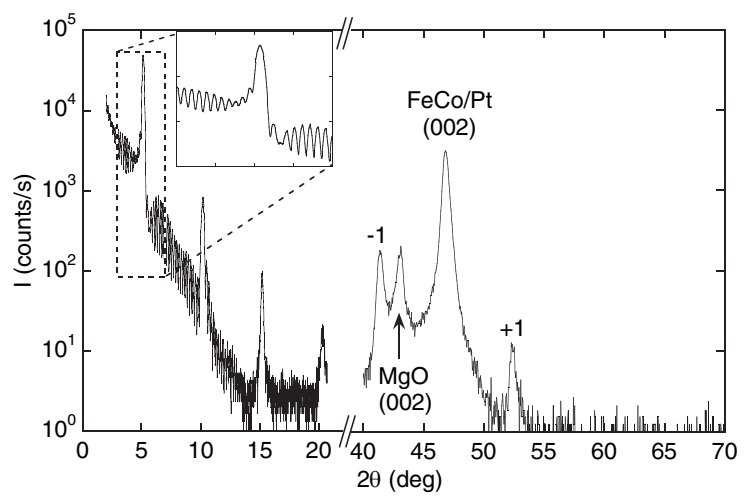

FIG. 2. X-ray reflectivity and diffraction, with an enlargement (inset) around the first chemical modulation peak showing interference fringes from the total thickness. Note the break on the $2 \theta$ scale. 
WINGIXA [16] and found to be in good agreement with the nominal values. The simulations also yielded an interface width of $1 \mathrm{ML}$, which is the lower limit for any real sample created through sputtering or other physical vapor deposition techniques [13]. Given the above assumptions, the error introduced in the $c / a$ ratio is estimated to be on the order of \pm 0.03 . The tetragonal distortion nevertheless falls in the immediate vicinity of the predicted high- $K_{\mathrm{u}}$ region, $1.2-1.25$.

The in-plane magnetization $M_{\|}$was measured as a function of the applied field $H$ at $40 \mathrm{~K}$ and at $300 \mathrm{~K}$ using a superconducting quantum interference device (SQUID) magnetometer. The obtained $M_{\|}(H)$ curves are displayed in Fig. 3. The perpendicular $M_{\perp}(H)$ loops, shown in Fig. 3, were measured using the polar magneto-optic Kerr effect (PMOKE). The saturation magnetization, obtained from the SQUID measurements, was $\mu_{0} M_{\mathrm{s}}=2.52 \mathrm{~T}$ at $40 \mathrm{~K}$ and $\mu_{0} M_{\mathrm{s}}=2.27 \mathrm{~T}$ at $300 \mathrm{~K}$. These values were used to scale the relative PMOKE data. The room temperature inplane magnetization was also measured with longitudinal MOKE (LMOKE), which showed that the $M_{\|}(H)$ loops had negligible width. However, the LMOKE signal quality was severely impaired due to the reduction of 1 order of magnitude compared to the polar effect [17], and the data are not included in Fig. 3. Only the PMOKE and SQUID

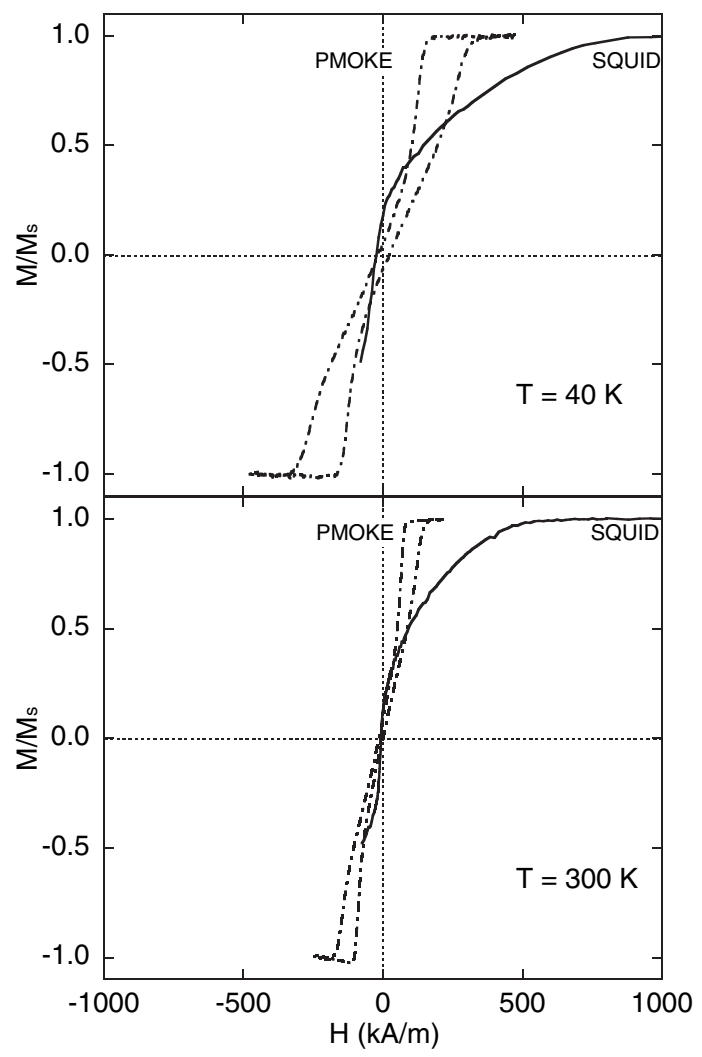

FIG. 3. Upper panel: normalized magnetization data at $40 \mathrm{~K}$ obtained from SQUID (in-plane, II ) and polar MOKE (perpendicular, $\perp$ ). Lower panel: the corresponding data at $300 \mathrm{~K}$. loops were used for the calculation of $K_{\mathrm{u}}$, as described below.

The average area between the in-plane and out-of-plane magnetization curves yields the effective MAE, $K_{\text {eff }}=$ $K_{\mathrm{u}}-\mu_{0} M_{\mathrm{s}}^{2} / 2$, where the last term is the magnetostatic shape anisotropy [18]. The latter, which competes with $K_{\mathrm{u}}$ and tends to align the magnetization in the plane of the film, amounts to $2.5 \mathrm{MJ} / \mathrm{m}^{3}$ at $40 \mathrm{~K}$. From these data a very large experimental $K_{\mathrm{u}}=(2.9 \pm 0.2) \mathrm{MJ} / \mathrm{m}^{3}$ is obtained at $40 \mathrm{~K}$. Using $a_{\mathrm{FeCo}}=0.268 \mathrm{~nm}$ and $c_{\mathrm{FeCo}}=$ $0.317 \mathrm{~nm}$ to calculate the volume of the FeCo unit cell yields a moment of $2.5 \mu_{\mathrm{B}} /$ atom and $K_{\mathrm{u}}=(208 \pm$ 14) $\mu \mathrm{eV} /$ atom in FeCo, which is of the same order of magnitude as the predicted $K_{\mathrm{u}}$ at low temperature. The corresponding values at $300 \mathrm{~K}$ are $2.2 \mu_{\mathrm{B}} /$ atom and (153 \pm 2) $\mu \mathrm{eV} /$ atom, respectively, and the magnetization retains its perpendicular direction at room temperature.

To compare the experimental findings with the previous calculations on bulk bet Fe-Co [4] we show in Fig. 4 the experimental room temperature $K_{\mathrm{u}}$ values for the superlattice series [12] together with cuts along three different Co concentrations in the theoretical plot [4] of $K_{\mathrm{u}}$ versus composition and $c / a$. The agreement is fair, considering the difference in temperature and the generally observed discrepancies between theoretical and experimental MAE values. The correct direction of the easy axis and the order of magnitude of the MAE is in general obtained, but the exact values of $K_{\mathrm{u}}$ can differ by as much as $50 \%$ [6]. To

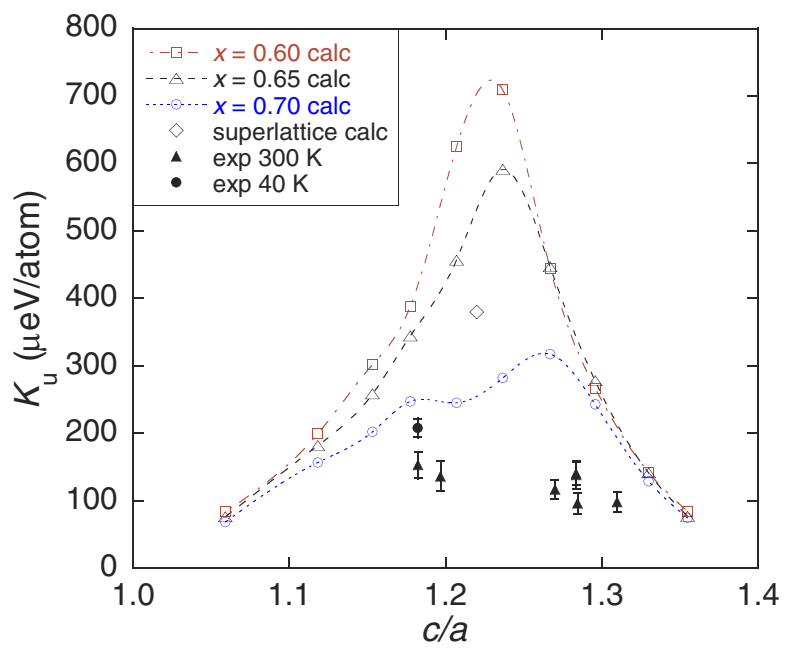

FIG. 4 (color online). Theoretical zero-temperature intrinsic MAE vs bct $c / a$ in the bulk alloys $\mathrm{Fe}_{1-x} \mathrm{Co}_{x}$ with $x=0.60$, 0.65 , and 0.70 (curves), compared to the room temperature experimental values for the superlattices with $(64 \pm 5)$ at. \% Co (solid triangles). The curves are guides to the eye. The experimental value for the " $3 / 7$ " sample $(c / a=1.18 \pm 0.03)$ at $40 \mathrm{~K}$ is included (solid circle), as well as the theoretical result for the corresponding structure with perfect interfaces (diamond). Further details on the samples with $c / a>1.18$ will be published elsewhere [12]. 
estimate the influence of temperature we have added the $40 \mathrm{~K}$ data for the " $3 / 7$ " sample in the same figure.

As the previous calculations $[4,6]$ were performed for bulk Fe-Co alloys, and since the resulting MAE is extremely dependent on the Fe-Co $c / a$ ratio [4], we performed additional first-principles calculations of $K_{\mathrm{u}}$ and $M_{\mathrm{s}}$ using the structure, with perfect interfaces, of the sample shown in Fig. 1. The calculations were done using a fully relativistic implementation of the full-potential linear muffin tin orbitals (FP-LMTO) method [19], modeling the Fe-Co alloy within the virtual crystal approximation [4]. Since the perpendicular lattice parameters of individual layers are difficult to extract experimentally, we performed a structural relaxation of the superlattice structure sketched in Fig. 1 using the plane wave VASP code $[20,21]$ with the all-electron projector augmented wave [22] method and the generalized gradient approximation, keeping the in-plane lattice parameter fixed at the experimental value. The calculated $c / a$ ratio of the relaxed alloy layers amounts to 1.22 , in fair agreement with the experimental value $(1.18 \pm 0.03)$ obtained from XRD. The relaxed structure was then used to calculate the zerotemperature $K_{\mathrm{u}}$ and $M_{\mathrm{s}}$ using the full-potential linear muffin tin orbitals code, giving $380 \mu \mathrm{eV} /$ atom and $2.29 \mu_{\mathrm{B}}$ /atom, respectively, in the FeCo layers. Hence, the calculations reproduce the important features of the low-temperature experiments: the perpendicular magnetization, the large MAE, and the size of the magnetic moment. Moreover, it was clear from a comparison of calculations performed with and without spin-orbit coupling in the Pt layers that this does not change the observed large $K_{\mathrm{u}}$. We note in passing that the large spin-orbit coupling of the Pt atom has in other materials been found to influence the MAE rather heavily $[23,24]$.

In our previous work [4] we pointed to the possibility of the MAE reaching some $700-800 \mu \mathrm{eV} /$ atom in Fe-Co alloys, provided the combined chemical and structural properties were optimized. The presently grown samples, with their given alloy composition, have structural parameters that lie somewhat outside the region where the maximum in $K_{\mathrm{u}}$ is predicted to occur, as revealed by our present experimental and theoretical data. This naturally opens up the possibility of further improvement of the materials properties of $\mathrm{Fe}-\mathrm{Co}$ alloys as recording media.

We gratefully acknowledge John M. Wills for supplying the FP-LMTO code, Ola Wilhelmsson for assistance with XPS, and financial support from the Swedish Research Council (VR), the Swedish Foundation for Strategic Research (SSF), the Royal Physiographic Society in Lund, the Göran Gustafsson Foundation, and Seagate Inc., Bloomington, USA. Parts of the calculations were performed at the High Performance Computing Center North (HPC2N), Sweden.

*Electronic address: gabriella.andersson@fysik.uu.se

[1] D. A. Thompson and J. S. Best, IBM J. Res. Dev. 44, 311 (2000).

[2] D. Weller and A. Moser, IEEE Trans. Magn. 35, 4423 (1999).

[3] D. Weller, A. Moser, L. Folks, M. E. Best, W. Lee, M. Toney, M. Schwickert, J.-U. Thiele, and M. Doerner, IEEE Trans. Magn. 36, 10 (2000).

[4] T. Burkert, L. Nordström, O. Eriksson, and O. Heinonen, Phys. Rev. Lett. 93, 027203 (2004).

[5] B. M. Lairson and B. M. Clemens, Appl. Phys. Lett. 63, 1438 (1993).

[6] T. Burkert, O. Eriksson, P. James, S. I. Simak, B. Johansson, and L. Nordström, Phys. Rev. B 69, 104426 (2004).

[7] K. Han, K. Yu-Zhang, H. Kung, J. D. Embury, B. J. Daniels, and B. M. Clemens, Philos. Mag. A 82, 1633 (2002).

[8] M. Sakurai, Phys. Rev. B 50, 3761 (1994).

[9] M. Sakurai, N. Imamura, K. Hirano, and T. Shinjo, J. Magn. Magn. Mater. 147, 16 (1995).

[10] B. J. Daniels, W. D. Nix, and B. M. Clemens, Appl. Phys. Lett. 66, 2969 (1995).

[11] V. Mathet, T. Devolder, C. Chappert, J. Ferré, S. Lemerle, L. Belliard, and G. Guentherodt, J. Magn. Magn. Mater. 260, 295 (2003).

[12] G. Andersson, M. Björck, C. Chacon, C. Zlotea, P. Warnicke, S. Valizadeh, B. Sanyal, and J. Ferré (to be published).

[13] P.F. Fewster, X-Ray Scattering from Semiconductors (Imperial College Press, London, 2003).

[14] J. Birch, J.-E. Sundgren, and P. F. Fewster, J. Appl. Phys. 78, 6562 (1995).

[15] C. Kittel, Introduction to Solid State Physics (Wiley, New York, 1996), 7th ed.

[16] D. K. G. de Boer, Phys. Rev. B 44, 498 (1991).

[17] Z. Q. Qiu and S. D. Bader, Rev. Sci. Instrum. 71, 1243 (2000).

[18] M. T. Johnson, P. J. H. Bloemen, F. J. A. den Broeder, and J. J. de Vries, Rep. Prog. Phys. 59, 1409 (1996).

[19] J. M. Wills, O. Eriksson, M. Alouani, and D. L. Price, in Electronic Structure and Physical Properties of Solids: The Uses of the LMTO Method (Springer, New York, 2000), pp. 148-167.

[20] G. Kresse and J. Hafner, Phys. Rev. B 47, R558 (1993).

[21] G. Kresse and J. Furthmüller, Phys. Rev. B 54, 11169 (1996).

[22] P. E. Blöchl, Phys. Rev. B 50, 17953 (1994).

[23] M. Cinal, J. Phys. Condens. Matter 13, 901 (2001).

[24] G. H. O. Daalderop, P. J. Kelly, and M.F. H. Schuurmans, Phys. Rev. B 50, 9989 (1994). 